\title{
Overexpression of the endoplasmic reticulum stress- inducible gene TIN1 causes abnormal pollen surface morphology in Arabidopsis
}

\author{
Yuji Iwata', Tsuneyo Nishino ${ }^{2}$, Nozomu Koizumi ${ }^{1 *}$ \\ ${ }^{1}$ Graduate School of Life and Environmental Sciences, Osaka Prefecture University, 1-1 Gakuen-cho, Naka-ku, Sakai, Osaka \\ 599-8531, Japan; ${ }^{2}$ Graduate School of Biological Sciences, Nara Institute of Science and Technology, 8916-5 Takayama-cho, \\ Ikoma, Nara 630-0192, Japan \\ *E-mail: nkoizumi@plant.osakafu-u.ac.jp Tel: +81-72-254-9424 Fax: +81-72-254-9416
}

Received August 17, 2017; accepted August 23, 2017 (Edited by T. Mizoguchi)

\begin{abstract}
The unfolded protein response (UPR) or the endoplasmic reticulum (ER) stress response occurs when folding and maturation of secretory and membrane proteins are impaired in the ER. The UPR induces a number of genes that encode ER-localized molecular chaperones and folding enzymes to increase folding capacity in the ER. We have identified Tunicamycin Induced 1 (TIN1), an Arabidopsis gene that is highly induced during the UPR. We have shown that TIN1 protein is localized in the ER but its physiological function remains to be elucidated. In the present study we generated and analyzed transgenic Arabidopsis plants expressing TIN1 under CaMV35S promoter to obtain insights into the physiological role of TIN1. We found that although TIN1-overexpressing plants grew as did wild-type plants under ambient laboratory conditions, their pollen grains exhibited abnormal surface morphology. The result suggests a specific role of TIN1 in secretion of proteins and/or lipids during pollen development.
\end{abstract}

Key words: Arabidopsis, endoplasmic reticulum, pollen, unfolded protein response.

Secretory and membrane proteins are synthesized on the endoplasmic reticulum (ER) in which folding and maturation of proteins occur before delivered to their final destinations via vesicle trafficking. When protein folding and maturation in the ER are perturbed or the amount of proteins loaded into the ER exceeds the capacity of the folding machinery in the ER, unfolded proteins accumulate in the ER. Under these so-called ER stress conditions, the unfolded protein response (UPR) or the ER stress response is initiated to induce transcriptional activation of genes encoding ER-resident molecular chaperones and folding enzymes to increase the protein folding capacity in the ER (Ron and Walter 2007).

In Arabidopsis, two transcription factors, bZIP60 and bZIP28, are activated during the UPR to induce transcription of genes encoding ER-resident molecular chaperones and folding enzymes (Howell 2013; Iwata and Koizumi 2012). bZIP60 is activated by cytoplasmic splicing, in which the removal of the 23-nt intron of bZIP60 mRNA causes a frameshift and results in the production of an active, nuclear form of bZIP60 (Nagashima et al. 2011). bZIP28 is a membrane-bound transcription factor activated by proteolytic processing within its transmembrane domain, which liberates the cytoplasmic portion of bZIP28 from the membrane (Iwata et al. 2017). Activation of bZIP60 and bZIP28 and transcriptional induction of genes encoding ER-resident molecular chaperones and folding enzymes have been observed during male gametophyte development (Iwata et al. 2008, 2010b) as well as in response to heat shock and pathogen attack (Gao et al. 2008; Nagashima et al. 2014; Wang et al. 2005), implicating a role of the UPR in these phenomena.

Polypeptides translated by ribosomes attached on the ER membrane enter the ER lumen and undergo folding and maturation (Ellgaard and Helenius 2003). BiP is an ER-resident heat shock protein 70 cognate and binds to nascent polypeptides to assist folding. Calreticulin and calnexin are molecular chaperones for proteins with $\mathrm{N}$-glycan chains. Genes encoding those molecular chaperones are induced during the UPR to increase the folding capacity in the ER under stress conditions (Iwata et al. 2010b; Martínez and Chrispeels 2003). Although their functions have been well known, there are also a number of ER stress-inducible genes whose functions remain to be characterized (Iwata et al. 2010b). Our transcriptomic analysis using tunicamycin, an inhibitor of $\mathrm{N}$-linked glycosylation that causes ER stress, identified

Abbreviations: ER, endoplasmic reticulum; SEM, scanning electron microscope; TIN1, Tunicamycin Induced 1; UPR, unfolded protein response. This article can be found at http://www.jspcmb.jp/

Published online September 16, 2017 
Tunicamycin Induced 1 (TIN1, AGI code At5g64510) (Iwata et al. 2010a, 2010b). We showed that TIN1 is induced in bZIP60-dependent manner during the UPR and that TIN1 encodes an ER-localized protein (Iwata et al. 2010a). We also showed that TIN1 is highly expressed in pollen grains and that the tin 1 mutant exhibits altered pollen surface structure, suggesting its role in pollen development and function (Iwata et al. 2012). In the present study, we generated and analyzed transgenic plants expressing TIN1 under CaMV35S promoter (hereafter referred to as TIN1-OX plants) to obtain deeper insights into the function of TIN1.

We used Arabidopsis thaliana ecotype Col-0 in this study. Plants were grown on soil in a growth chamber at $23^{\circ} \mathrm{C}$ under a 16 -h-light/8-h-dark cycle. For tunicamycin treatment, 10-day-old seedlings grown in half strength Murashige and Skoog medium supplemented with $1 \%$ sucrose were treated with $5 \mu \mathrm{g} \mathrm{ml}^{-1}$ tunicamycin or $0.1 \%$ dimethyl sulfoxide as a solvent control for $5 \mathrm{~h}$. To generate transgenic plants expressing TIN1 gene under CaMV35S promoter, TIN1 cDNA was PCR amplified using the primers TIN1-F-XbaI (5'-GTC TAG AAT GGG TCA CAG AGT ATT GGT TT-3') and TIN1-RPacI (5'-GTT AAT TAA TTA CAA GGT AAA AGG GCT TGG-3'), cloned into the pGEM-T Easy vector (Promega), and subcloned into the modified pBI121 vector, which harbors $\mathrm{XbaI}$ and $\mathrm{PacI}$ sites between CaMV35S promoter and Nos terminator. Transformation was carried out by the floral dip method (Clough and Bent 1998).

For quantitative RT-PCR (qRT-PCR), RNA was extracted from leaves of 4-week-old wild-type and TIN1$O X$ plants grown on soil and 10-day-old seedlings grown on half strength Murashige and Skoog medium using RNeasy Plant Mini Kit (Qiagen) and reverse transcribed using ExScript RT Reagent Kit (TaKaRa). Quantitative real-time PCR was carried out using SYBR Premix Ex Taq (TaKaRa) with LightCycler 480 Instrument (Roche). TIN1 and Act8 fragments were amplified using the following primers; TIN1-F-qPCR (5'-AAT ATG CGC CTT TCT TTA CCA T-3') and TIN1-R-qPCR (5'-GCA CCA TCT GCT AAT ATC ACT TTC-3') for TIN1, Act8F-qPCR ( $5^{\prime}$-TCA GCA CTT TCC AGC AGA TG-3') and Act8-R-qPCR (5'-ATG CCT GGA CCT GCT TCA T-3') for Act8. The expression level of TIN1 was normalized to that of Act8. The experiment was carried out with three biological replicates.

Mature pollen grains from wild-type and TIN1$O X$ plants were observed under a scanning electron microscope (SEM) as previously described (Iwata et al. 2012). In brief, mature pollen grains were placed on double-sided sticky carbon tape to the aluminum stub, which was then mounted on the cooling stage at $-20^{\circ} \mathrm{C}$. The specimen was observed with a SEM (S-3200N, Hitachi) at a chamber pressure of $30 \mathrm{~Pa}$ and an accelerating voltage of $15 \mathrm{kV}$.

A
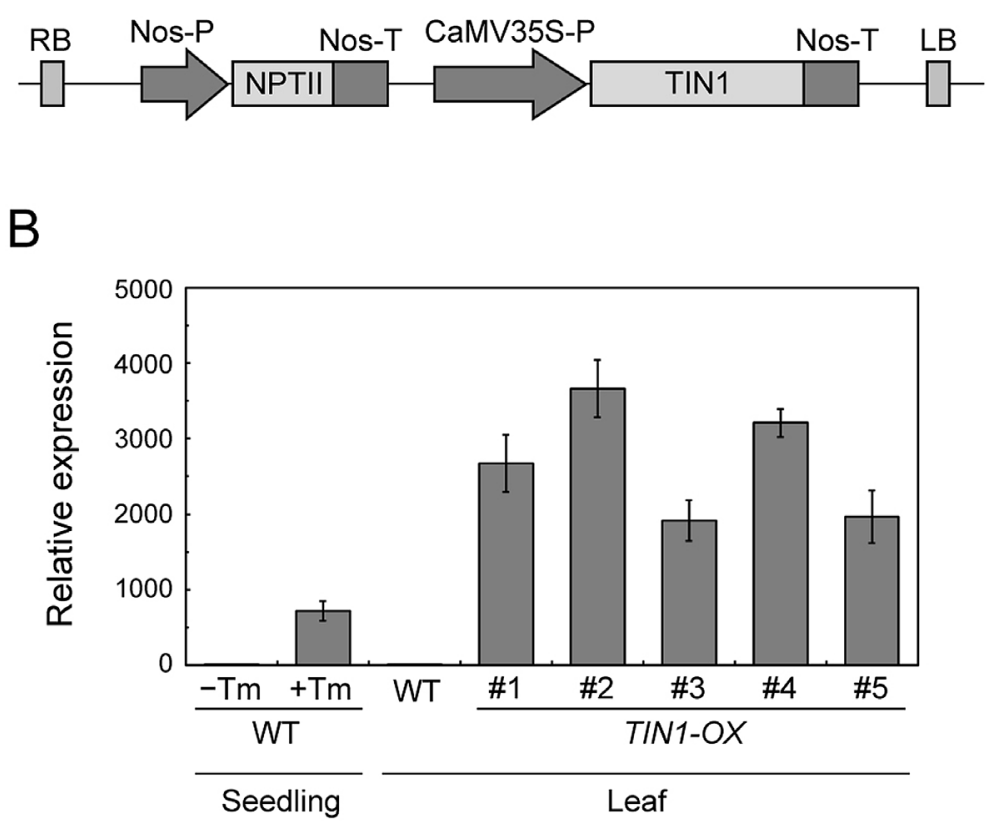

Figure 1. Generation of TIN1-OX plants. (A) A schematic diagram of the construct used to generate TIN1-OX plants. CaMV35S-P, CaMV35S promoter; Nos-P, Nos promoter; Nos-T, Nos terminator; NTPII, neomycin phosphotransferase II; RB, right border; LB, left border; (B) Expression level of TIN1 in wild-type and TIN1-OX plants. RNA was extracted from leaves of 4-week-old wild-type and TIN1-OX plants and subjected to quantitative RT-PCR analysis. Ten-day-old wild-type seedlings treated with $5 \mu \mathrm{g} \mathrm{ml}^{-1}$ tunicamycin (+Tm) or $0.1 \%$ dimethyl sulfoxide (-Tm) were also analyzed for comparison. The expression level of TIN1 was normalized to that of Act8. 
To generate TIN1-OX plants, we constructed a binary vector that expresses TIN1 gene under CaMV35S promoter (Figure 1A) and introduced it into Arabidopsis wild-type plants. We obtained five T3 lines that exhibit high TIN1 expression even without the ER stress inducer tunicamycin (Figure 1B). The expression level of TIN1 in TIN1-OX was comparable to that of tunicamycintreated wild-type plants (Figure 1B). When we grew wild-type and TIN1-OX plants side-by-side and compared the growth phenotype, TIN1-OX plants were indistinguishable from wild-type plants in growth and development, flower morphology, silique length, and seed size (Figure 2).

We have shown that TIN1 gene is highly expressed in pollen and that the tin1-1 mutant exhibits pollen grains with altered surface morphology (Iwata et al. 2012). We therefore observed pollen grains from wild-type and TIN1-OX plants under SEM and examined whether TIN1-OX plants have any defects in pollen grains. We found altered pollen grain morphology of TIN1-OX plants (Figure 3). We observed adherence of a substance on the pollen surface of TIN1-OX plants, which was not seen in that of wild-type plants.

We have shown that tin1-1 mutant plants also harbor pollen grains with abnormal surface structure but did not exhibit any defects in growth and development in ambient laboratory growth conditions (Iwata et al. 2012). The present study also demonstrated that the effect of TIN1 overexpression was confined to pollen grains. A possible explanation for this pollen-specific phenotypic alteration is that the role of TIN1 is to assist folding and maturation of pollen-specific secretory or membrane proteins, and altered levels of TIN1 affects the secretion of proteins and lipids, culminating in altered pollen surface morphology. A role of the UPR during male gametophyte development has been suggested by the previous observations that an Arabidopsis UPR-related mutant, ire1a ire1b, exhibit reduced male fertility (Deng et al. 2013, 2016). It is tempting to speculate that reduced TIN1 expression in the ire 1a ire1b mutant contributes to some extent to the reduced male fertility.

The UPR is conserved among diverse eukaryotic cells,
A

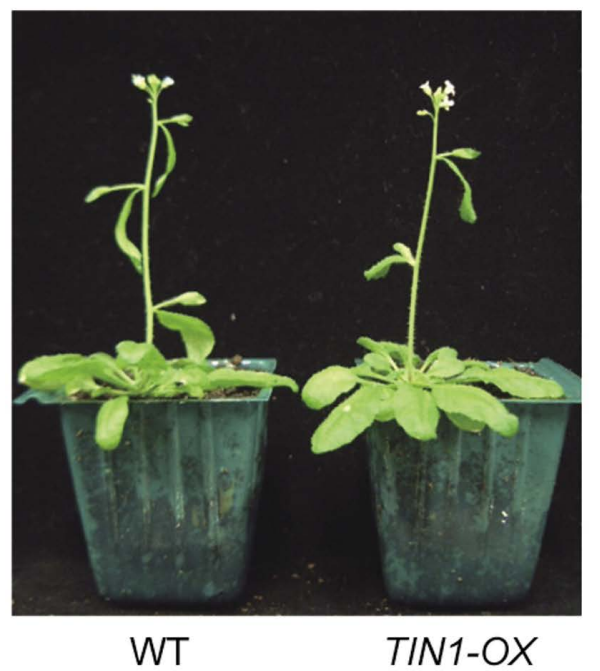

B

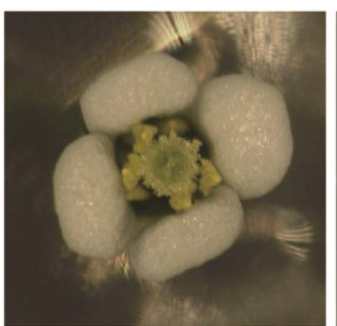

WT

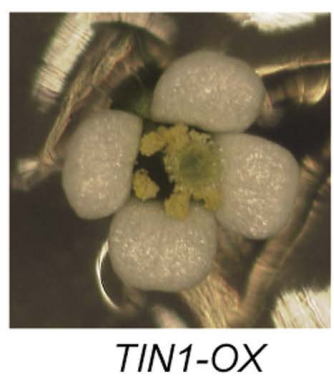

C

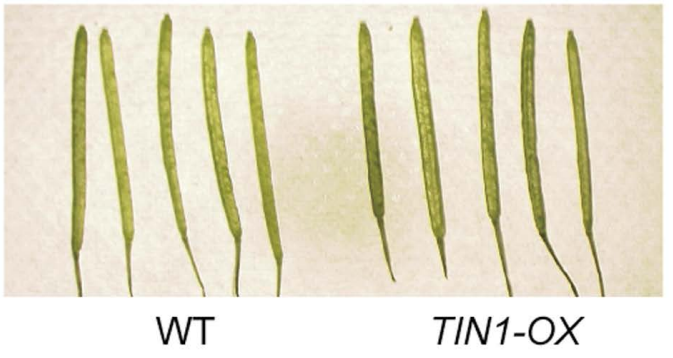

D

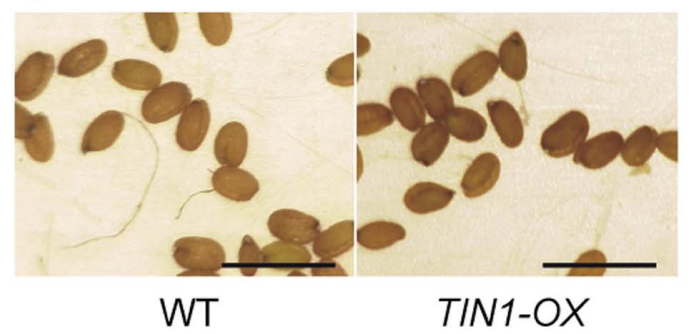

Figure 2. Observation of TIN1-OX plants. (A-D) Pictures of soil-grown wild-type and TIN1-OX (line \#2) plants. (A) Five-week-old plants. (B) Eight-week-old flowers. (C) Eight-week-old siliques. (D) Ten-week-old seeds. Bar=1 mm. 


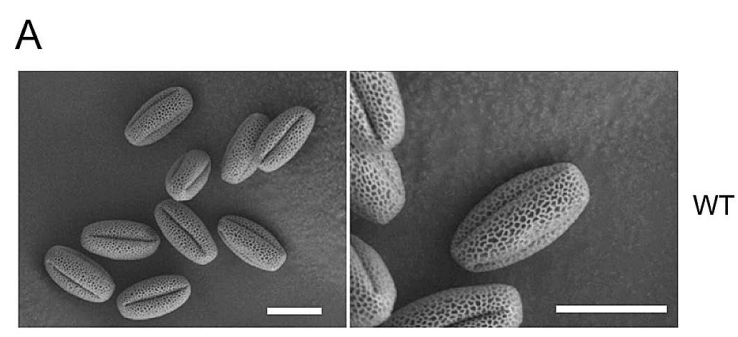

B

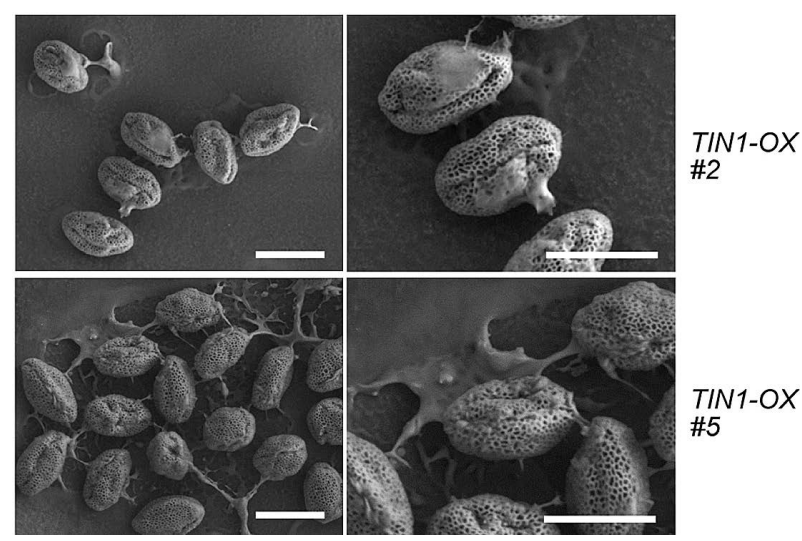

Figure 3. SEM observation of pollen grains of TIN1-OX plants. (A) SEM images of pollen grains of wild-type plants. (B) SEM images of pollen grains of TIN1-OX plants. Images from two independent TIN1$O X$ lines ( $\# 2$ and $\# 5$ ) were shown. $B a r=20 \mu \mathrm{m}$.

including yeast, animals, and plants (Howell 2013; Iwata and Koizumi 2012; Ron and Walter 2007). Although the molecular mechanism of transcription factor activation differ to some extent among different species (Iwata and Koizumi 2012), the UPR induces a similar set of genes, which encode ER molecular chaperones such as BiP and calnexin, to increase the protein folding capacity in the ER. However, although TIN1 is an ER stress-inducible gene that encodes an ER-localized protein (Iwata et al. 2010a), TIN1 homologs are found in sequenced genomes of plant species but not in those of other species. Elucidation of the function of TIN1 during pollen development would provide interesting insights into the diversity of the UPR and the ER folding machinery.

\section{Acknowledgements}

We thank Drs. Seiji Takayama (The University of Tokyo) and Megumi Iwano (Osaka University) for assistance in generating transgenic plants and taking SEM images. This work was supported by JSPS KAKENHI Grant Number 20380188 to N.K.

\section{References}

Clough SJ, Bent AF (1998) Floral dip: A simplified method for
Agrobacterium-mediated transformation of Arabidopsis thaliana. Plant J 16: 735-743

Deng Y, Srivastava R, Howell SH (2013) Protein kinase and ribonuclease domains of IRE1 confer stress tolerance, vegetative growth, and reproductive development in Arabidopsis. Proc Natl Acad Sci USA 110: 19633-19638

Deng Y, Srivastava R, Quilichini TD, Dong H, Bao Y, Horner HT, Howell SH (2016) IRE1, a component of the unfolded protein response signaling pathway, protects pollen development in Arabidopsis from heat stress. Plant J 88: 193-204

Ellgaard L, Helenius A (2003) Quality control in the endoplasmic reticulum. Nat Rev Mol Cell Biol 4: 181-191

Gao H, Brandizzi F, Benning C, Larkin RM (2008) A membranetethered transcription factor defines a branch of the heat stress response in Arabidopsis thaliana. Proc Natl Acad Sci USA 105: 16398-16403

Howell SH (2013) Endoplasmic reticulum stress responses in plants. Annu Rev Plant Biol 64: 477-499

Iwata Y, Ashida M, Hasegawa C, Tabara K, Mishiba K, Koizumi N (2017) Activation of the Arabidopsis membrane-bound transcription factor bZIP2 8 is mediated by site- 2 protease, but not site-1 protease. Plant J 91: 408-415

Iwata Y, Fedoroff NV, Koizumi N (2008) Arabidopsis bZIP60 is a proteolysis-activated transcription factor involved in the endoplasmic reticulum stress response. Plant Cell 20: 3107-3121

Iwata Y, Koizumi N (2012) Plant transducers of the endoplasmic reticulum unfolded protein response. Trends Plant Sci 17: $720-727$

Iwata Y, Nishino T, Iwano M, Takayama S, Koizumi N (2012) Role of the plant-specific endoplasmic reticulum stress-inducible gene TIN1 in the formation of pollen surface structure in Arabidopsis thaliana. Plant Biotechnol 29: 51-56

Iwata Y, Nishino T, Takayama S, Koizumi N (2010a) Characterization of a plant-specific gene induced by endoplasmic reticulum stress in Arabidopsis thaliana. Biosci Biotechnol Biochem 74: 2087-2091

Iwata Y, Sakiyama M, Lee MH, Koizumi N (2010b) Transcriptomic response of Arabidopsis thaliana to tunicamycin-induced endoplasmic reticulum stress. Plant Biotechnol 27: 161-171

Martínez IM, Chrispeels MJ (2003) Genomic analysis of the unfolded protein response in Arabidopsis shows its connection to important cellular processes. Plant Cell 15: 561-576

Nagashima Y, Iwata Y, Ashida M, Mishiba K, Koizumi N (2014) Exogenous salicylic acid activates two signaling arms of the unfolded protein response in Arabidopsis. Plant Cell Physiol 55: 1772-1778

Nagashima Y, Mishiba K, Suzuki E, Shimada Y, Iwata Y, Koizumi N (2011) Arabidopsis IRE1 catalyses unconventional splicing of bZIP60 mRNA to produce the active transcription factor. Sci Rep 1: 29

Ron D, Walter P (2007) Signal integration in the endoplasmic reticulum unfolded protein response. Nat Rev Mol Cell Biol 8: 519-529

Wang D, Weaver ND, Kesarwani M, Dong X (2005) Induction of protein secretory pathway is required for systemic acquired resistance. Science 308: 1036-1040 